\title{
Gene expression profiling reveals overexpression of TSPAN13 in prostate cancer
}

\author{
JOSE M. ARENCIBIA ${ }^{1}$, SUSANA MARTÍN ${ }^{1}$, FRANCISCO J. PÉREZ-RODRÍGUEZ ${ }^{2}$ and ANA BONNIN ${ }^{1}$ \\ ${ }^{1}$ Universidad Francisco de Vitoria, Facultad de CC. Biosanitarias, Departamento de Bioquímica y Biotecnología, \\ Pozuelo de Alarcón, 28223 Madrid; ${ }^{2}$ Hospital Universitario de Madrid-Monteprincipe, \\ Departamento de Anatomía Patológica, Boadilla del Monte, 28660 Madrid, Spain
}

Received August 28, 2008; Accepted October 21, 2008

DOI: 10.3892/ijo_00000170

\begin{abstract}
Prostate cancer is one of the most frequent malignancies in the Western world. The identification of additional molecular markers is needed to refine the diagnosis of prostate cancer and to develop more effective therapies. In order to identify molecular abnormalities involved in prostate cancer progression, we performed gene expression analysis of prostate cancer samples compared to matched normal tissue from the same patient using a cancer-related microarray. Amplified RNA was hybridized to a cDNA microarray containing 6386 genes and tissue microarrays were used to study protein expression levels. Using significance analysis of microarrays, we identified $>1300$ genes differentially expressed in prostate cancer compared to normal tissue. Forty-two of these genes were highly upregulated in prostate cancer while 169 were highly repressed. We found that the gene coding for tspan 13 was upregulated $>2$-fold in $75 \%$ of the samples analyzed. Immunohistochemistry analysis of prostate cancer tissue microarrays showed that tspan 13 is overexpressed in $80 \%$ of prostate cancer samples analyzed. We found that tspan13 expression inversely correlates with Gleason score $(\mathrm{p}=0.01)$ and PSA preoperative levels $(\mathrm{p}=0.11)$ and directly correlates with presence of prostatic intraepithelial neoplasia in tumor tissue $(\mathrm{p}=0.04)$. Moreover, we detected tspan 13 expression in low-grade prostatic intraepithelial neoplasia. Thus, our results show that tspan 13 is overexpressed in prostate cancer and its expression correlates with factors of favourable outcome. Therefore we suggest that tspan 13 may have an important role in the progression of prostate cancer.
\end{abstract}

Correspondence to: Dr Jose M. Arencibia, Universidad Francisco de Vitoria, Facultad de CC. Biosanitarias, Departamento de Bioquímica y Biotecnología, Ctra. M-515 Pozuelo-Majadahonda, Km 1,800, 28223 Pozuelo de Alarcón, Madrid, Spain E-mail: j.arencibia@ufv.es

Key words: immunohistochemistry, tetraspanin, prostatic intraepithelial neoplasia, $\alpha$-methylacyl coenzyme A racemase, microarray, tumor progression

\section{Introduction}

Prostate cancer is the most frequent malignancy diagnosed in European men and the third leading cause of male cancerrelated deaths in Europe (1). At present, the treatment of prostate cancer involves surgery, radiotherapy, chemotherapy and/or hormonal therapy. These therapies are potentially curative for patients with clinically localized disease, although a significant proportion of them will ultimately relapse. Treatment with androgen ablation therapy of patients who failed primary curative attempts is rarely effective and the vast majority eventually develops hormone-refractory disease which is almost uniformly fatal. Similarly, there is not an effective treatment for those patients who have advanced stage and/or metastatic disease at the time of diagnosis. Thus, there is a need to develop more effective therapies and the finding of potential therapeutic targets as well as predictors of treatment response is required.

Prostate-specific antigen (PSA) screening has led to the identification of many men with localized cancer when therapy is likely to be effective, although elevated PSA levels occur also in other inflammatory or obstructive uropathies, lowering the specificity of PSA as a cancer marker (2). Furthermore, PSA also lacks the sensitivity to detect early-stage tumors, since a large fraction of men with a normal serum PSA level have biopsy-proven prostate cancer (3). Therefore, identification of additional molecular markers is needed to refine the diagnosis of prostate cancer. In addition, the heterogeneous behaviour of prostatic neoplasms requires the discovery of novel biomarkers that will allow to discriminate a slow growing cancer from a more aggressive one with potential to metastasize.

Cancer-associated molecular and genetic alterations are being revealed by using various strategies including DNA microarray technology. Gene expression profiling of normal and tumor tissue from individuals with different types of cancer is currently used to identify new prognostic biomarkers, to predict response to therapy and/or to discover molecular targets for the development of novel therapeutic approaches. Using this methodology many genes showing altered expression patterns have been identified in malignant tissues. Particularly, microarray studies of prostate cancer have led to the identification of genes that are differentially expressed in tumor cells and genes whose expression correlates with tumor 
grade, metastasis and disease recurrence (4-9). Moreover, using high-throughput molecular analysis several novel prostate cancer markers have been proposed in the past few years, including hepsin (7), $\alpha$-methylacyl coenzyme A racemase $(A M A C R)(10)$, enhancer of zeste homolog 2 (Drosophila) (11) and cell surface associated mucin 1 (5). Some of them, such as AMACR and hepsin, are currently undergoing validation as diagnostic tests for prostate cancer. These discoveries have also provided the rationale for the development of new therapies, such as the immunotherapeutic approaches that are being sought using mucin 1 (12).

In this study, we performed gene expression analysis of prostate cancer samples compared to matched normal tissue from the same patient using a cancer-related microarray in order to identify molecular abnormalities involved in prostate cancer progression that can be used as markers of disease and/or as targets for novel therapies.

\section{Materials and methods}

cDNA microarray. Prostate cancer samples and adjacent normal prostate samples were provided by the Barcelona Hospital Clinic tumor bank (IDIBAPS). The study was approved by the Research Committee of the Universidad Francisco de Vitoria and the Ethics Committee of the Barcelona Hospital Clinic. Pathological data of the samples are shown in Table I. Total RNA was extracted, as previously described (13). Purity and quality of the samples were analyzed by agarose gel electrophoresis. Total RNA $(5 \mu \mathrm{g})$ was used to generate cDNA with the SuperScript ${ }^{\mathrm{TM}}$ choice system for cDNA synthesis (Invitrogen SA, Barcelona, Spain). Doublestranded cDNA was purified by phenol/chlorophorm followed by ethanol precipitation and subsequently used as template for T7 RNA polymerase amplification using the MEGAscript ${ }^{\circledR}$ T7 kit (Ambion, Austin, TX, USA). Amplified RNA (aRNA) from normal prostate was labelled using cyanine 3-conjugated dUTP (Amersham, Piscataway, NJ, USA) while aRNA from tumor samples was labelled with cyanine 5-conjugated dUTP (Amersham). Labelled aRNA mixture $(30 \mu \mathrm{g})$ was hybridized to the CNIO Human Oncochip 1.1c (http://bioinfo.cnio.es/ data/oncochip/) composed of 6386 related cancer genes. Slides were scanned and quantified, as previously described (13).

Initial data analysis was performed using the DNMAD and the preprocessor modules of the Gene Expression Profile Analysis Suite v3.1 (GEPAS; http://www.gepas.org) (14). GenePix files were uploaded into the DNMAD module and normalized using print tip loess without background substraction and flagged points were returned as missing values. The file obtained was submitted to the preprocessor and replicates on the array were averaged after eliminating inconsistent replicates (threshold from the median 0.5). Missing values were imputted using the KNN method $(\mathrm{K}=15)$ after removing those patterns with $>4$ missing values. The data obtained was analyzed using TIGR MeV (http://www.tm4. org/) (15). Multiple testing to detect differentially expressed genes was carried out using the significance analysis of microarrays (SAM) method (16), using one class analysis and allowing for 1,000 random permutations. Genes identified as statistically significant with SAM were further classified using
Table I. Pathological data of the samples used for cDNA microarray.

\begin{tabular}{lll}
\hline Sample no. & Gleason score & \multicolumn{1}{c}{ pTN } \\
\hline PCA01 & $6(3+3)$ & pT3aN0 \\
PCA02 & $7(3+4)$ & pT2cN0 \\
PCA04 & $7(3+4)$ & pT2c \\
PCA05 & $7(3+4)$ & pT2cN0 \\
PCA06 & $6(2+4)$ & pT2c \\
PCA07 & $9(4+5)$ & pT2bN0 \\
PCA08 & $7(4+3)$ & pT3a \\
PCA09 & $7(3+4)$ & pT3b \\
PCA10 & $5(3+2)$ & pT2cN0 \\
PCA11 & $7(4+3)$ & pT2cN0 \\
PCA12 & $6(3+3)$ & pT2cN1 \\
PCA13 & $6(2+4)$ & pT2cN0 \\
PCA14 & $7(3+4)$ & pT3aN0 \\
PCA15 & $7(3+4)$ & pT2c \\
PCA16 & $7(3+4)$ & pT3a \\
PCA17 & $6(3+3)$ & pT2c \\
\hline
\end{tabular}

the self organizing tree algorithm (SOTA) (17). The data discussed in this publication have been deposited in NCBIs Gene Expression Omnibus (GEO, http://www.ncbi.nlm.nih. gov/geo/) and are accessible through GEO Series accession number GSE9347. Oncomine analysis were performed using Oncomine 3.0, an online database which allows users to query differential expression results for a gene of interest across collected microarray data sets (18).

Quantitative RT-PCR ( $q R T-P C R)$. RNA expression analysis by qRT-PCR was performed on an ABI PRISM 7900 HT (Applied Biosystems, Foster City, CA, USA) using SYBRGreen PCR master mix (Applied Biosystems). Total RNA (500 ng) was reverse transcribed and PCR was performed using the Taq Man ${ }^{\circledR}$ gene expression assays (Applied Biosystems) for tumor-associated calcium signal transducer 1 (TACSTD1), glycine-N-acyltransferase-like 1 (GLYATL1) and TSPAN13. Human glyceraldehyde-3-phosphate dehydrogenase was used as internal control.

Tissue microarray. The antibody used in this study was raised in rabbit against a peptide corresponding to amino acids 116-128 in the large extracellular region of the tspan 13 protein (sequence accession number: NP_055214) (Pacific Immunology, San Diego, CA, USA). Accumax tissue microarrays (TMA) containing stage II and III prostate cancer cores (Biomol GmbH, Hamburg, Germany) were used. The slides containing the histological core sections were deparaffinized in xylene, rehydrated in graded ethanol $(100-80 \%)$, washed in distilled water and then boiled for $20 \mathrm{~min}$ in $10 \mathrm{mM}$ sodium citrate buffer $(\mathrm{pH}$ 6.0) containing $0.05 \%$ Tween-20 for antigen retrieval. Sections were blocked with $2 \%$ goat serum in $0.05 \mathrm{M}$ Tris- $\mathrm{HCl} \mathrm{pH} 7.6$ containing $0.9 \% \mathrm{NaCl}$ and $0.05 \%$ Tween-20 at room temperature and 


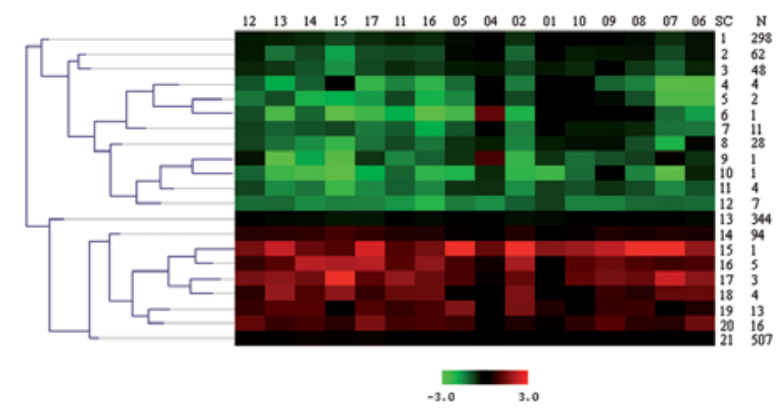

Figure 1. SOTA dendrogram generated by TIGR MeV of significant expressed genes obtained after SAM analysis in 16 prostate cancer samples. Numbers on top of the dendrogram correspond to each prostate cancer analyzed. Each line in the dendrogram corresponds to a SOTA cluster where genes with similar expression patterns are grouped. Numbers on the right correspond to the cluster number and the number of genes included in each cluster. The band under the dendrogram shows the intensity levels of the resultant cluster vectors, which is represented by green and red heat map colouring. Red corresponds to the highest expression level, black corresponds to moderate expression and green to the lowest expression. The horizontal branch length on the left reflects the degree of variability between clusters. SC, SOTA cluster number and $\mathrm{N}$, number of genes included in each cluster.

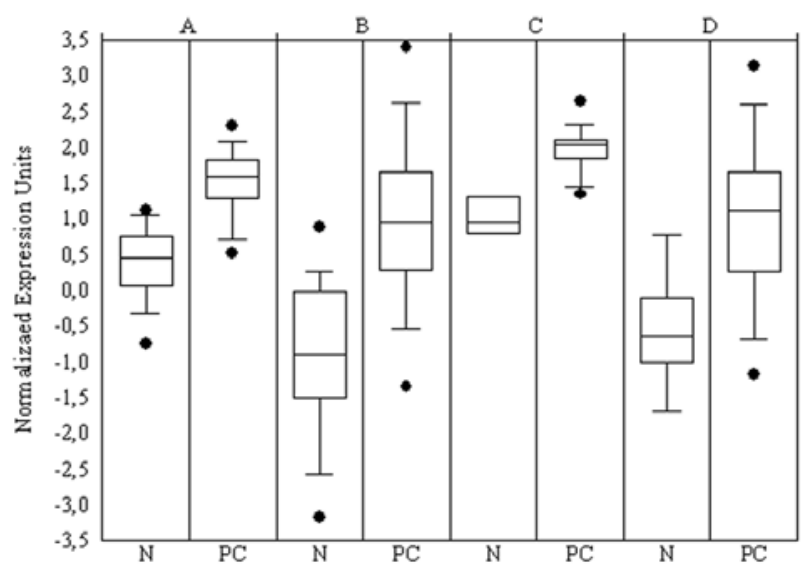

Figure 2. Box plots of normalized expression units for TSPAN13 in prostate cancer (PC) relative to normal/benign prostate tissue $(\mathrm{N})$ of four different microarray analysis of prostate cancer processed by Oncomine 3.0. Lower and upper line of the box represent 25th and 75th percentiles. Median value is represented by the line within the box. Error bars above and below the box indicate the 90th and 10th percentiles. Dots above and below the box represent the maximum and the minimum values. Data originally obtained by A, Lapointe et al (5); B, Tomlins et al (36); C, Vanaja et al (38) and D, Dhanasekaran et al (37).

then incubated with rabbit anti-tspan13 polyclonal antibody at 1:25 dilution or prediluted rabbit anti-AMACR polyclonal antibody (ABCAM, Cambridge, UK) overnight at $4^{\circ} \mathrm{C}$. After blocking endogenous peroxidase with $3 \% \mathrm{H}_{2} \mathrm{O}_{2}$, bound antibody was detected with Rabbit ExtrAvidin ${ }^{\circledR}$ Peroxidase staining kit (Sigma, St. Louis, MO, USA) according to the manufacturer's instructions and visualized using 3-amino-9ethylcarbazole as a chromogenic substrate. Slides were counterstained using Mayer's haematoxylin. Specificity of labelling was confirmed by both omission of the primary antibody and preincubation of the primary antibody with the immunizing peptide. The intensity of staining was graded semiquantitatively and each tissue core was assigned a score on a scale from 0 to 3 , designated as 0 (negative), 1 (weak), 2 (moderate) and 3 (strong). Then, duplicate cores from each patient were averaged before statistical analysis. Digital images were taken using NIS-Elements image processing software with a Zeiss Axiophot microscope coupled to a Nikon DS-2Mv digital camera.

Statistics. Statistical analysis was performed using SSPS software (SPSS Inc. Chicago, IL, USA). Bivariate correlation was used to assess potential significant associations between prognostic clinicopathological parameters and tspan 13 or AMACR protein expression. Therefore, the staining intensity from duplicate cores from the same patient was averaged and samples that scored $<1$ were considered negative. Values are expressed as mean \pm SD. All statistical tests were two-sided and a $\mathrm{p}<0.05$ was considered statistically significant.

\section{Results}

Gene expression profiles in prostate cancer samples. We analyzed the expression levels of genes in prostate cancer tissue by hybridization of aRNA to a cDNA microarray representing $>6000$ cancer-related genes. After one class SAM analysis, 1454 sequences corresponding to 1358 unique genes were found to be significantly expressed in prostate cancer at a $\Delta$ value threshold of 2 with a median of false discovery rate (FDR) of $0.077 \%$ (data not shown). Using SOTA, these genes were further classified in 21 clusters divided in two main arms of centroids vectors in the dendrogram corresponding to genes under- and overexpressed in prostate cancer compared to normal prostate tissue (Fig. 1). Most of the genes (94\%) whose expression varied slightly across all the samples analyzed were classified into six clusters while clusters number 6, 9, 10 and 15 included only one gene, being activating transcription factor 3 (ATF3), complement component $7(C 7)$, osteoglycin $(O G N)$ and $A M A C R$, respectively. Forty-two highly induced genes were classified in clusters 15 to 20 in the lower arm of the dendrogram while 169 highly repressed genes where grouped in clusters 4 to 12 (Fig. 1). Among highly induced genes, $A M A C R$ was overexpressed $>2$-fold in all the samples analyzed $(100 \%)$ and was classified as a single gene cluster after SOTA analysis, whereas overexpression of TACSDT1 was found in $87.5 \%$ of the samples and GLYATL1, golgi membrane protein 1 (GOLM1) and TSPAN13 in 75\% of the samples. TSPAN13 and GLYATL1 were classified by SOTA analysis in cluster number 17 together with fructose-1,6-bisphosphatase 1 (FBPl). Among highly repressed genes, only two genes encoding for pleckstrin homology-like domain, family A, member 2 (PHLDA2) and for eukaryotic translation initiation factor 3, subunit A (EIF3A) were underexpressed $>2$-fold in all the samples analyzed, while several genes such as osteoglicin $(O G N)$, tumor protein p73-like (TP73L) or annexin A1 (ANXA1) were downregulated in $81.2,68.7$ and $62.5 \%$ of the samples, respectively.

Gene expression changes observed by microarray analysis were confirmed by qRT-PCR for selected genes. RNA expression levels for TACSTD1, TSPAN13 and GLYATL1 in prostate cancer compared to normal tissue determined by qRT-PCR did not vary from those obtained in the microarray analysis (not shown). In addition, we used Oncomine to interrogate publicly available prostate cancer expression array data sets. As shown in Fig. 2, TSPAN13 is significantly 


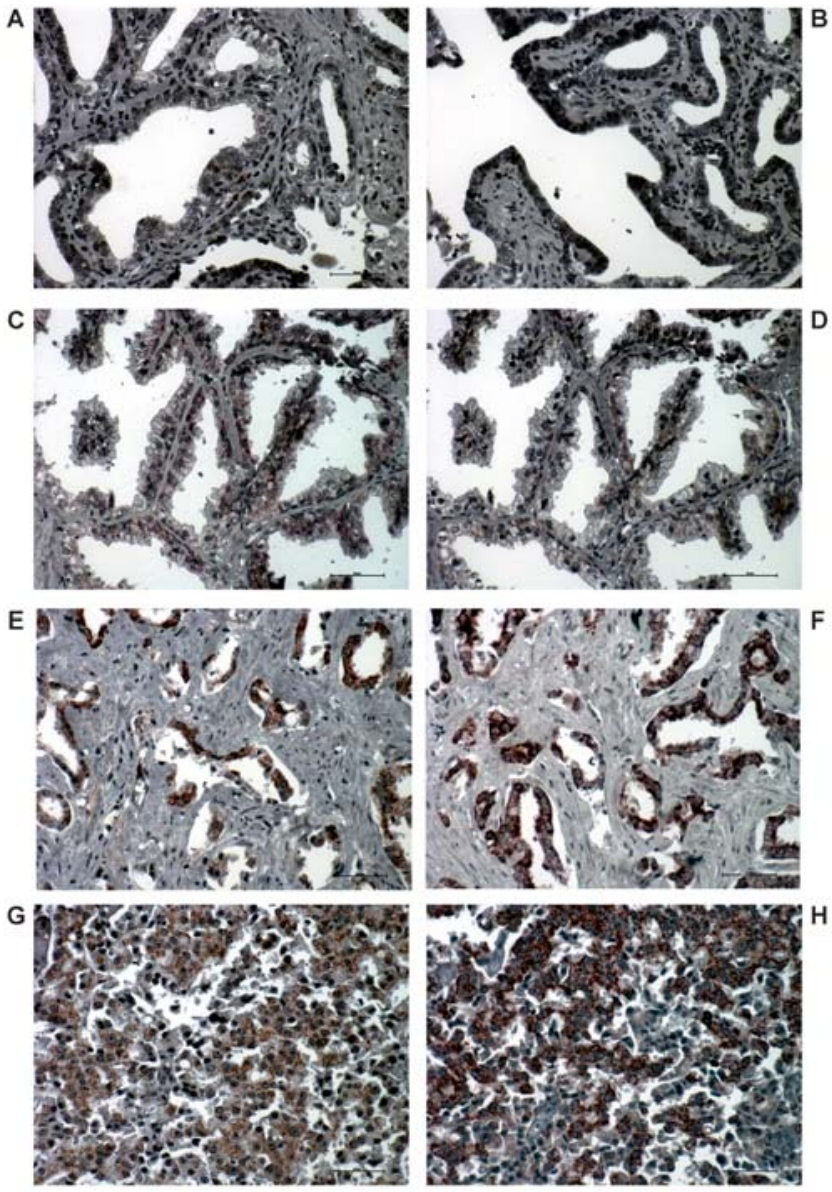

Figure 3. Immunohistochemical staining of prostate tissue using anti-tspan13 (A, C, E and G) or anti-AMACR polyclonal antibodies (B, D, F and H). Representative images for normal prostate (A and B), low-grade PIN (C and D), prostate cancer GS 7 (E and F) and GS $9(\mathrm{G}$ and $\mathrm{H})$ are shown. Bar in the bottom-right corner of each image indicates magnification $(50 \mu \mathrm{m})$.

overexpressed in prostate cancer compared to normal prostate tissue. Consistent with our analysis, Oncomine also showed significant differential expression in prostate cancer compared with normal tissue for upregulated genes, such as $A M A C R$, TACSTD1 and GOLM1, as well as downregulated ones such as EIF3A, OGN, TP73L or ANXA1 (not shown).

Immunohistochemistry. To study protein levels of tspan 13 and AMACR in prostate cancer samples, we performed immunohistochemistry analysis using prostate cancer TMA (Fig. 3). Pathological grade of tissue samples contained in TMA slides varied from Gleason score (GS) 6 to 10 , although most represented samples were GS 7 (34 patients) and GS 9 (39 patients) and only one corresponded to GS 10. In total, we analyzed 175 cores from 88 prostate cancer patients. Specific immunostaining was achieved using the polyclonal antibody generated against tspan 13 since no staining was observed in negative controls (Fig. 4). The two cores from the same patient representing GS 10 were negative for both tspan 13 and AMACR and therefore were not included in the analysis (not shown). In total, 80.7 and $78.4 \%$ of the patients did express tspan 13 and AMACR proteins, respectively, with an average staining score of $1.73 \pm 0.64$ for tspan13 and $1.88 \pm 0.78$ for AMACR.
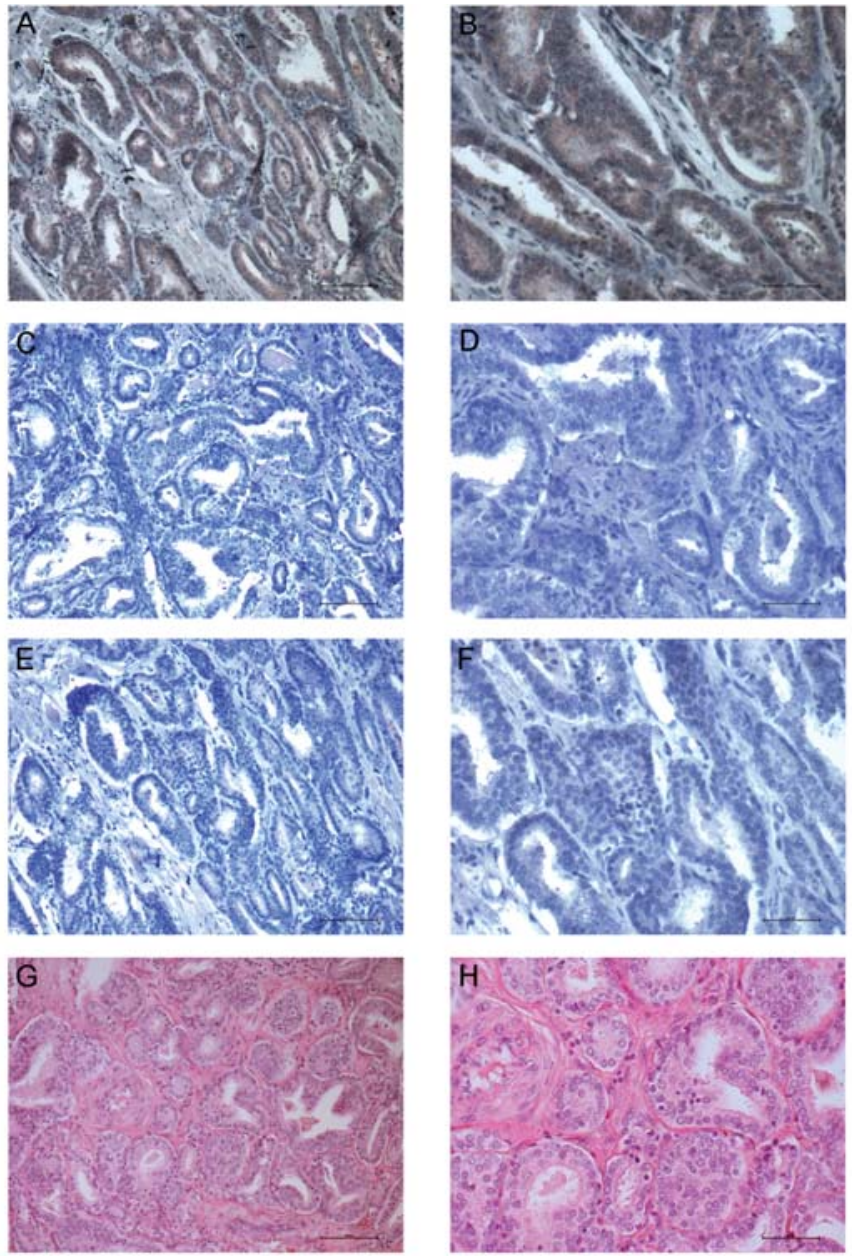

Figure 4. Prostate cancer tissue immunostaining using anti-tspan 13 polyclonal antibody (A and B). Specificity of antibody binding to tspan13 was confirmed by omitting primary antibody $(\mathrm{C}$ and $\mathrm{D})$ or by preincubating the primary antibody with the immunizing peptide (E and $F$ ). Haematoxylin-eosin staining $(\mathrm{G}$ and $\mathrm{H})$. Bar in the bottom-right corner of the figure indicates magnification: $100 \mu \mathrm{m}(\mathrm{A}, \mathrm{C}, \mathrm{E}$ and $\mathrm{G}) ; 50 \mu \mathrm{m}(\mathrm{B}, \mathrm{D}, \mathrm{F}$ and $\mathrm{H}$ )

Expression of tspan13 was inversely correlated with GS $(\mathrm{p}=0.01)$ since staining for tspan 13 decreased from $1.75 \pm 0.96$ in prostate cancer patients of GS 6 to $1.28 \pm 0.87$ in GS 9. Similarly, staining intensity for tspan 13 was also inversely correlated with preoperative PSA levels, although it was not statistically significant $(\mathrm{p}=0.11)$. Direct correlation between tspan 13 expression and presence of prostatic intraepithelial neoplasia (PIN) in tumor tissue was also significant $(\mathrm{p}=0.04)$ (Table II). On the contrary, AMACR staining showed no significant correlation with GS, PIN or PSA preoperative levels (Table II).

The TMA used in this study included 8 cores representing non-neoplastic tissue from 8 prostate cancers patients. Upon pathological examination, only two of these cores were normal prostate tissue, while the rest corresponded to lowgrade PIN. Tspan13 immunostaining was positive in all the PIN samples and negative in normal tissue while AMACR immunostaining was positive in only four of the PIN samples (Fig. 3). Average staining of these samples was $0.63 \pm 0.52$ for tspan 13 and $0.25 \pm 0.71$ for AMACR. 
Table II. Correlation between clinicopathological factors and tspan13 or AMACR immunostaining.

\begin{tabular}{|c|c|c|c|c|}
\hline \multirow[b]{2}{*}{ GS } & \multicolumn{2}{|c|}{$\begin{array}{c}\text { Percentage of } \\
\text { positive samples }\end{array}$} & \multicolumn{2}{|c|}{$\begin{array}{l}\text { Average staining } \\
\text { intensity }\end{array}$} \\
\hline & Tspan13 & AMACR & Tspan13 & AMACR \\
\hline 6 & 100.00 & 75.00 & $1.75 \pm 0.96$ & $1.63 \pm 1.38$ \\
\hline 7 & 88.24 & 91.18 & $1.68 \pm 0.77$ & $1.63 \pm 0.81$ \\
\hline 8 & 90.91 & 81.82 & $1.32 \pm 0.46$ & $1.41 \pm 0.70$ \\
\hline 9 & 69.23 & 66.67 & $1.28 \pm 0.87$ & $1.44 \pm 1.10$ \\
\hline $\mathrm{Pc}^{\mathrm{a}}$ & & & -0.26 & -0.12 \\
\hline $\mathrm{p}^{\mathrm{b}}$ & & & 0.01 & 0.23 \\
\hline \multicolumn{5}{|l|}{$\begin{array}{l}\text { PSA } \\
(\mathrm{ng} / \mathrm{ml})\end{array}$} \\
\hline $0-10$ & 80.39 & 76.47 & $1.54 \pm 0.86$ & $1.49 \pm 0.94$ \\
\hline $10-20$ & 88.00 & 88.00 & $1.46 \pm 0.67$ & $1.66 \pm 0.85$ \\
\hline$>20$ & 66.67 & 66.67 & $1.13 \pm 0.68$ & $1.33 \pm 1.04$ \\
\hline $\mathrm{Pc}^{\mathrm{a}}$ & & & -0.17 & $\mathrm{nc}$ \\
\hline $\mathrm{p}^{\mathrm{b}}$ & & & 0.11 & - \\
\hline \multicolumn{5}{|l|}{ PIN } \\
\hline Negative & 65.71 & 65.71 & $1.27 \pm 0.86$ & $1.41 \pm 1.12$ \\
\hline Positive & 90.57 & 86.79 & $1.58 \pm 0.75$ & $1.58 \pm 0.82$ \\
\hline $\mathrm{Pc}^{\mathrm{a}}$ & & & 0.21 & 0.11 \\
\hline $\mathrm{p}^{\mathrm{b}}$ & & & 0.04 & 0.31 \\
\hline
\end{tabular}

GS, Gleason score; AMACR, $\alpha$-methylacyl coenzyme A racemase; PSA, prostate-specific antigen; PIN, prostatic intraepithelial

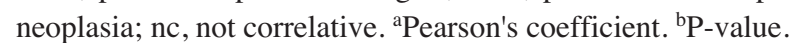

\section{Discussion}

We generated expression profiles of 16 prostate cancers using the CNIO Human Oncochip 1.1c to identify differentially expressed genes that can be potentially used as diagnostic or prognostic tools, as well as targets for new therapeutic approaches. Using SAM, we found $>1300$ genes significantly expressed in prostate cancer compared to adjacent normal tissue. Because most of these genes varied slightly among all the samples analyzed, we used SOTA to further classify significant genes in clusters of highly repressed and highly expressed genes. SOTA provides the possibility of reducing number of gene patterns obtained by microarray analysis to a scale in which differences and similarities among patterns can be easily analyzed. Also, it allows the management of the resolution with which the system is analyzed producing dendograms that describe the system at different hierarchical levels (17).

We found that $>2 / 3$ of highly deregulated genes in our study were downregulated. Tumor suppressor proteins are usually lost during cancer progression and underexpression or loss of gene expression in tumor cells compared to normal tissue has been used to identify tumor suppressor genes. Among the genes that we found to be downregulated in prostate cancer, only PHLDA2 and EIF3A gene expression levels were $>2$-fold lower than in normal tissue in all the samples analyzed.

The PHLDA2 gene is located in 11p15.5, an imprinted region in chromosome 11 predicted to harbour several tumor suppressor genes and it has been suggested that PHLDA2 may play a role in negatively regulating tissue growth by participating in programmed cell death (19). Thus, PHLDA2 could be considered as novel tumor suppressor protein in prostate cancer, and the decreased level of PHLDA2 expression that we detected may be involved in prostate cancer progression, although further studies are necessary to confirm its function in prostate cancer.

The other gene that we found downregulated in all samples analyzed, EIF3A, codes for the largest subunit of eukaryotic translation initiation factor eIF3. Altered eIFs function can influence protein synthesis and therefore cause abnormal cell growth and malignant transformation. Further studies will determine whether the reduced expression that we found for EIF3A in prostate cancer may correlate with disease prognosis and/or progression.

Several genes that we observed to be upregulated in our study have been previously related with prostate cancer progression and it has been suggested their use as potential markers for the detection of cancer or as targets for new therapeutic approaches for the treatment of prostate cancer. Among the highly expressed genes, we found that AMACR gene was overexpressed in all the samples analyzed. We have also confirmed AMACR protein overexpression in a large number of prostate cancer samples by immunohistochemistry analysis using TMA, with $78.4 \%$ of the cases analyzed showing positive staining. Our results are therefore in agreement with those obtained previously by other groups $(10,20,21)$. These studies also support the use of AMACR to confirm the presence of prostate cancer in histological sections. Moreover, its combination with novel basal marker antigens such as p63 has been suggested to aid in the detection of difficult cases $(22,23)$. Even though, AMACR staining should be interpreted with caution, since its expression in nonneoplastic prostate is age-related (24) and it has significant limitations in morphologically difficult prostate cancer (25).

Another gene widely overexpressed in our study is TACSTD1, a carcinoma associated antigen also known as Ep-CAM (epithelial cell adhesion molecule). This protein functions as a homophilic cell-cell adhesion molecule with cadherin-modulating properties which is involved in epithelial cell proliferation and tumor progression (26). TACSTD1 has been shown to be overexpressed in several types of cancers such as breast (27), ovarian (28) and prostate (29) and monoclonal antibodies designed to bind TACSTD1 have been tested in phase I trials in patients with advanced prostate cancer $(30,31)$.

Among the highly expressed genes, TSPAN13 was overexpressed $>2$-fold compared to normal prostate in $75 \%$ of the samples analyzed. TSPAN13 gene codes for a membrane protein that belongs to the transmembrane 4 superfamily of proteins, also known as the tetraspanin family. Based on protein sequence similarities there are at least 33 putative family members in humans (UniProt Knowledgebase Release 12.8) (32) characterized by their four transmembrane regions, 
two extracellular and one intracellular loops, and two short Nand C-terminal cytoplasmic domains. Although the function of most members of the family is currently unknown, a relevant feature of the tetraspanins is their potential to associate with other transmembrane proteins forming tetraspanin-enriched microdomains providing a scaffold for the transmission of external stimuli to intracellular signalling components $(33,34)$. Our present results are in agreement with several microarray studies that have found TSPAN13 overexpression in prostate cancer samples (5,35-38). Moreover, TSPAN13 has been included in a 'feature' representing genes more highly expressed in prostate adenocarcinoma (5). Together with TSPAN13, other genes that we found by cDNA microarray analysis to be overexpressed in prostate cancer such as AMACR, TACSTD1, FBP1 (fructose-1,6-bisphosphatase 1) and GOLMI (Golgi membrane protein 1) have been also included in this 'feature' (5).

Oncomine analysis shows that TSPAN13 is also significantly overexpressed in mucinous, clear cell and endometrioid adenocarcinomas of the ovary (39), breast cancer $(40,41)$, testicular germ cell tumors (42-44), bladder carcinoma (45) and B-cell acute lymphoblastic leukaemia (46). In breast cancer, TSPAN13 gene overexpression is related with oestrogen and progesterone receptor status being significantly higher in oestrogen receptor and progesterone receptor positive breast cancers $(40,41)$. Presence of oestrogen and/or progesterone receptor is associated with favourable prognosis in breast cancer. Furthermore, expression of TSPAN13 was found by microarray analysis to be associated with a less aggressive phenotype of breast cancer cells (47). In addition, it has been suggested recently that tspan13 acts as a tumor suppressor protein in breast cancer, since transfection of a breast cancer cell line that expressed low levels of mRNA for tspan13 with a vector containing TSPAN13 fused to GFP inhibited cell growth and invasion (48).

In our study, we show that overexpression of tspan 13 mRNA in prostate cancer is correlated with an increase of protein level in cancer tissue. We found that $80.7 \%$ of the patients analyzed were positive for tspan 13 and that the staining intensity was inversely correlated with GS. Higher Gleason patterns (GS 8-10), usually associated with unfavourable prognosis and with a higher metastasic potential, showed decreased level of tspan13 expression than lower Gleason patterns (GS 6-7). Similarly, we found an inverse correlation between tspan 13 expression and preoperative PSA levels which are associated with the interval to biochemical progression after radical prostatectomy (49). Moreover, patients with high Gleason score and high PSA levels had shorter progression-free survival after radical prostatectomy $(50,51)$. Since the frequency of tspan 13 overexpression decreased in higher Gleason patterns and higher preoperative PSA levels, it seems likely that tspan 13 may play a protective role in prostate cancer.

We found that tspan13 is expressed also in PIN lesions that usually accompany prostate cancer in histological tissue sections. It will be important to determine the presence of tspan13 protein in other preneoplastic lesions such as highgrade PIN and proliferative inflammatory atrophy as well as in benign prostatic hyperplasia. Future studies will address these issues as well as will focus on the biological function of tspan13 in prostate cancer tumorigenesis. Although known members of the tetraspanin family are involved in signal transduction events related with cell motility, the function of tspan 13 in prostate cancer is not known. Whether tspan 13 behaves as a tumor metastasis suppressor in prostate cancer as some other members of the tetraspanin family like CD82/ Kail (52) has to be determined with additional studies. Further insights into the role that tspan 13 plays in prostate cancer progression may provide a rationale for its use as a diagnostic tool to detect early stage prostate cancers. Also, the predicted membrane localization of tspan 13 makes it an attractive target for immunotherapy approaches, as is the case of TACSTD1 or MUC1.

In conclusion, using gene expression analysis we found that the gene TSPAN13 is upregulated in prostate cancer samples compared with matching normal tissue. We also confirmed that tspan 13 protein is overexpressed in a high percentage of prostate cancer samples by tissue microarrays and its expression correlates with factors of favourable outcome for prostate cancer. Therefore, tspan 13 may have an important role in the progression of prostate cancer.

\section{Acknowledgements}

We are grateful to Dr A. Dopazo and Dr L. Lombardia for help with cDNA microarrys and to R. Alvarez and G. Luengo for technical assistance. This study was supported by grants from Comunidad Autonoma de Madrid (08.1/0002.1/2001) and Universidad Francisco de Vitoria.

\section{References}

1. Ferlay J, Autier P, Boniol M, Heanue M, Colombet M and Boyle P: Estimates of the cancer incidence and mortality in Europe in 2006. Ann Oncol 18: 581-592, 2007.

2. Brawer MK: Prostate-specific antigen. Semin Surg Oncol 18: 3-9, 2000.

3. Thompson IM, Pauler DK, Goodman PJ, et al: Prevalence of prostate cancer among men with a prostate-specific antigen level $<$ or =4.0 ng per milliliter. N Engl J Med 350: 2239-2246, 2004.

4. Dhanasekaran SM, Barrette TR, Ghosh D, et al: Delineation of prognostic biomarkers in prostate cancer. Nature 412: 822-826, 2001.

5. Lapointe J, Li C, Higgins JP, et al: Gene expression profiling identifies clinically relevant subtypes of prostate cancer. Proc Natl Acad Sci USA 101: 811-816, 2004.

6. Luo J, Duggan DJ, Chen Y, et al: Human prostate cancer and benign prostatic hyperplasia: molecular dissection by gene expression profiling. Cancer Res 61: 4683-4688, 2001.

7. Magee JA, Araki T, Patil S, et al: Expression profiling reveals hepsin overexpression in prostate cancer. Cancer Res 61: 5692-5696, 2001

8. Singh D, Febbo PG, Ross K, et al: Gene expression correlates of clinical prostate cancer behavior. Cancer Cell 1: 203-209, 2002.

9. Welsh JB, Sapinoso LM, Kern SG, et al: Large-scale delineation of secreted protein biomarkers overexpressed in cancer tissue and serum. Proc Natl Acad Sci USA 100: 3410-3415, 2003.

10. Xu J, Stolk JA, Zhang X, et al: Identification of differentially expressed genes in human prostate cancer using subtraction and microarray. Cancer Res 60: 1677-1682, 2000.

11. Varambally S, Dhanasekaran SM, Zhou M, et al: The polycomb group protein EZH2 is involved in progression of prostate cancer. Nature 419: 624-629, 2002.

12. Li Y and Cozzi PJ: MUC1 is a promising therapeutic target for prostate cancer therapy. Current Cancer Drug Targets 7: 259, 2007

13. Arencibia JM, Del Rio M, Bonnin A, Lopes R, Lemoine NR and Lopez-Barahona M: Doxazosin induces apoptosis in LNCaP prostate cancer cell line through DNA binding and DNAdependent protein kinase down-regulation. Int J Oncol 27: $1617-1623,2005$ 
14. Montaner D, Tarraga J, Huerta-Cepas J, et al: Next station in microarray data analysis: GEPAS. Nucleic Acids Res 34: W486-W491, 2006.

15. Saeed AI, Sharov V, White J, et al: TM4: a free, open-source system for microarray data management and analysis. Biotechniques 34: 374-378, 2003.

16. Tusher VG, Tibshirani R and Chu G: Significance analysis of microarrays applied to the ionizing radiation response. Proc Nat Acad Sci USA 98: 5116-5121, 2001

17. Herrero J, Valencia A and Dopazo J: A hierarchical unsupervised growing neural network for clustering gene expression patterns. Bioinformatics 17: 126-136, 2001.

18. Rhodes DR, Yu J, Shanker K, et al: ONCOMINE: a cancer microarray database and integrated data-mining platform. Neoplasia 6: 1-6, 2004.

19. Qian N, Frank D, O'Keefe D, et al: The IPL gene on chromosome $11 \mathrm{p} 15.5$ is imprinted in humans and mice and is similar to TDAG51, implicated in Fas expression and apoptosis. Hum Mol Genet 6: 2021-2029, 1997.

20. Luo J, Zha S, Gage WR, et al: \{alpha\}-Methylacyl-CoA Racemase: A new molecular marker for prostate cancer. Cancer Res 62: 2220-2226, 2002.

21. Rubin MA, Zhou M, Dhanasekaran SM, et al: alpha-Methylacy coenzyme A racemase as a tissue biomarker for prostate cancer. JAMA 287: 1662-1670, 2002.

22. Molinie V, Fromont G, Sibony M, et al: Diagnostic utility of a p63/alpha-methyl-CoA-racemase (p504s) cocktail in atypical foc in the prostate. Mod Pathol 17: 1180-1190, 2004.

23. Hameed O, Sublett J and Humphrey PA: Immunohistochemical stains for p63 and alpha-methylacyl-CoA racemase, versus a cocktail comprising both, in the diagnosis of prostatic carcinoma: a comparison of the immunohistochemical staining of 430 foci in radical prostatectomy and needle biopsy tissues. Am J Surg Pathol 29: 579-587, 2005.

24. Gologan A, Bastacky S, McHale T, Yu J, Cai C, MonzonBordonaba F and Dhir R: Age-associated changes in alpha-methy CoA racemase (AMACR) expression in nonneoplastic prostatic tissues. Am J Surg Pathol 29: 1435-1441, 2005.

25. Varma M and Jasani B: Diagnostic utility of immunohistochemistry in morphologically difficult prostate cancer: review of current literature. Histopathology 47: 1-16, 2005.

26. Litvinov SV, Balzar M, Winter MJ, et al: Epithelial cell adhesion molecule (Ep-CAM) modulates cell-cell interactions mediated by classic cadherins. J Cell Biol 139: 1337-1348, 1997.

27. Gastl G, Spizzo G, Obrist P, Dunser M and Mikuz G: Ep-CAM overexpression in breast cancer as a predictor of survival. Lancet 356: 1981-1982, 2000.

28. Kim JH, Herlyn D, Wong KK, et al: Identification of epithelia cell adhesion molecule autoantibody in patients with ovarian cancer. Clin Cancer Res 9: 4782-4791, 2003.

29. Poczatek RB, Myers RB, Manne U, Oelschlager DK, Weiss HL, Bostwick DG and Grizzle WE: Ep-Cam levels in prostatic adenocarcinoma and prostatic intraepithelial neoplasia. J Urol 162: 1462-1466, 1999 .

30. de Bono JS, Tolcher AW, Forero A, et al: ING-1, a monoclonal antibody targeting Ep-CAM in patients with advanced adenocarcinomas. Clin Cancer Res 10: 7555-7565, 2004.

31. Oberneder R, Weckermann D, Ebner B, et al: A phase I study with adecatumumab, a human antibody directed against epithelial cell adhesion molecule, in hormone refractory prostate cancer patients. Eur J Cancer 42: 2530-2538, 2006.

32. Gasteiger E, Gattiker A, Hoogland C, Ivanyi I, Appel RD and Bairoch A: ExPASy: The proteomics server for in-depth protein knowledge and analysis. Nucleic Acids Res 31: 3784-3788, 2003.

33. Hemler ME: Tetraspanin proteins mediate cellular penetration, invasion, and fusion events and define a novel type of membrane microdomain. Annu Rev Cell Dev Biol 19: 397-422, 2003.

34. Levy $\mathrm{S}$ and Shoham T: The tetraspanin web modulates immune-signalling complexes. Nat Rev Immunol 5: 136-148, 2005.
35. Halvorsen OJ, Oyan AM, Bo TH, et al: Gene expression profiles in prostate cancer: association with patient subgroups and tumour differentiation. Int J Oncol 26: 329-336, 2005.

36. Tomlins SA, Mehra R, Rhodes DR, et al: Integrative molecular concept modeling of prostate cancer progression. Nat Genet 39: 41, 2007.

37. Dhanasekaran SM, Dash A, Yu J, et al: Molecular profiling of human prostate tissues: insights into gene expression patterns of prostate development during puberty. FASEB J 04-2415fje, 2004.

38. Vanaja DK, Cheville JC, Iturria SJ and Young CYF: Transcriptional silencing of zinc finger protein 185 identified by expression profiling is associated with prostate cancer progression. Cancer Res 63: 3877-3882, 2003.

39. Hendrix ND, Wu R, Kuick R, Schwartz DR, Fearon ER and Cho KR: Fibroblast growth factor 9 has oncogenic activity and is a downstream target of Wnt signaling in ovarian endometrioid adenocarcinomas. Cancer Res 66: 1354-1362, 2006.

40. Chin K, DeVries S, Fridlyand J, et al: Genomic and transcriptional aberrations linked to breast cancer pathophysiologies. Cancer Cell 10: 529-541, 2006.

41. Minn AJ, Gupta GP, Siegel PM, et al: Genes that mediate breast cancer metastasis to lung. Nature 436: 518-524, 2005.

42. Korkola JE, Houldsworth J, Chadalavada RS, et al: Downregulation of stem cell genes, including those in a $200-\mathrm{kb}$ gene cluster at $12 \mathrm{p} 13.31$, is associated with in vivo differentiation of human male germ cell tumors. Cancer Res 66: 820-827, 2006.

43. Skotheim RI, Lind GE, Monni O, et al: Differentiation of human embryonal carcinomas in vitro and in vivo reveals expression profiles relevant to normal development. Cancer Res 65: 5588-5598, 2005

44. Sperger JM, Chen X, Draper JS, et al: Gene expression patterns in human embryonic stem cells and human pluripotent germ cell tumors. Proc Natl Acad Sci USA 100: 13350-13355, 2003.

45. Sanchez-Carbayo M, Socci ND, Lozano J, Saint F and Cordon-Cardo C: Defining molecular profiles of poor outcome in patients with invasive bladder cancer using oligonucleotide microarrays. J Clin Oncol 24: 778-789, 2006.

46. Andersson A, Ritz C, Lindgren D, et al: Microarray-based classification of a consecutive series of 121 childhood acute leukemias: prediction of leukemic and genetic subtype as well as of minimal residual disease status. Leukemia 21: 1198-1203, 2007.

47. Huang H, Groth J, Sossey-Alaoui K, Hawthorn L, Beall S and Geradts J: Aberrant expression of novel and previously described cell membrane markers in human breast cancer cell lines and tumors. Clin Cancer Res 11: 4357-4364, 2005.

48. Huang H, Sossey-Alaoui K, Beachy SH and Geradts J: The tetraspanin superfamily member NET-6 is a new tumor suppressor gene. J Cancer Res Clin Oncol 133: 761-769, 2007.

49. Gonzalez CM, Roehl KA, Antenor JV, Blunt LW, Han M and Catalona WJ: Preoperative PSA level significantly associated with interval to biochemical progression after radical retropubic prostatectomy. Urology 64: 723, 2004.

50. Bastian PJ, Gonzalgo ML, Aronson WJ, et al: Clinical and pathologic outcome after radical prostatectomy for prostate cancer patients with a preoperative Gleason sum of 8 to 10 Cancer 107: 1265-1272, 2006

51. Rodriguez-Covarrubias F, Larre S, De La Taille A, Abbou C-C and Salomon L: The outcome of patients with pathological Gleason score $>$ or $=8$ prostate cancer after radical prostatectomy. BJU Int 101: 305-307, 2008.

52. Dong JT, Lamb PW, Rinker-Schaeffer CW, Vukanovic J, Ichikawa T, Isaacs JT and Barrett JC: KAI1, a metastasis suppressor gene for prostate cancer on human chromosome 11p11.2. Science 268: 884-886, 1995 